\title{
HISTOLOGICAL AND IMMUNOHISTOCHEMICAL EVALUATION OF THE POSSIBLE EFFECTS OF DIFFERENT DOSES OF CAFFEINE ON SUBMANDIBULAR SALIVARY GLAND OF ADULT MALE ALBINO RATS USING (ANNEXIN A5) MARKER
}

\author{
Afaf Khalaf Ali*, Amr Helmy Elbolok* and Doha Saber Mohamed **
}

\begin{abstract}
Background :Caffeine is one of the most widely consumed substances in the world. The main sources of caffeine in society are coffee or tea The cytotoxicity of caffeine may be due to its ability to trigger apoptosis

Material and methods :A total number of 30 adult male albino rats (150-200 g) were used and were divided randomly into five equal groups ( $n=6)$ control group: 6 rats were I.P injected with sterile: Group II \& IIa :12 rats were I. P. injected with single daily dose $(2.5 \mathrm{mg} / 100 \mathrm{~g}$ body weight/day) for three months this dose is equivalent to minimum human dose. Group IIa: 6 rats stopped treatment and left for one month after the period of treatment.Group III \& IIIa: 12 rats were I.P. Injected with single daily dose $(10 \mathrm{mg} / 100 \mathrm{~g}$ body weight/day) for three months this dose is equivalent to maximum human dose. Group IIIa: 6 rats stopped treatment and left for one month after the period of treatment Samples from the submandibular salivary glands were fixed in $10 \%$ buffered neutral formalin and prepared routinely for paraffin sectioning and staining for histopathological changes and immuno- histochemical investigations of apoptotic cell antigen (Annexin A5).
\end{abstract}

Results : Histopathological examination of caffeine treated rats revealed marked changes in glandular architecture, the acini became shrunkn with loss of their rnormal circular arrangement and their cytoplasm was vacuolated. Some serous cells degenerated and replaced by eosinophlic material. The nuclear changes appeared in form of (pleomorphism, hypertrophy, hyperchromatism and pyknosis), numerous normal and abnormal mitotic Figures were detected. Intercalated and excretory ducts were dilated., blood vessels were dilated and congested. The immuno expression of Annexin A5 in the cytoplasmic basement membrane of the ductal cells was intense and highly significant. Changes were more aggressive in (group III). Recovery of some histological changes were observed especially in group received lower dose of caffeine .

Conclusion: Excessive use of Caffeine markedly affect the histological structure of submandibular salivary gland. These changes could be reversible after caffeine stoppage if the doses is small while the histopathological changes of the gland is irreversible if the doses is large .

KEY WARDS: Caffeine, Submandibular gland, Annexin A5 .

* Department of Oral Biology, Faculty of Dentistry, Minia University, Egypt.

** Department of Histology and Cell Bilogy, Faculty of Medicine, Sohag University, Egypt 


\section{INTRODUCTION}

Caffeine is one of the most widely consumed substances in the world. The main sources of caffeine in society are coffee or tea which constitute upwards of $90 \%$ of standard intake in society with the remaining 10\% coming from cocoa products these numbers were before the advent of energy drinks and mainstream supplementation, however. More recent data show that coffee + tea dropped to $83 \%$ to accommodate the rise of soda to $12 \%$ of intake in the United States ${ }^{[1]}$ Caffeine is added to food and drink items as well as to certain medications to relieve drowsiness or enhance the effects of pain killers whether caffeine is consumed in food or as a medicine it changes the way your brain and body work and changes how you behave and feel. ${ }^{[2]}$ The cytotoxicity of caffeine may be due to its ability to trigger apoptosis A number of cysteine proteases, known as caspases, play important roles in apoptosis similar to members of the Bcl-2 family which regulate mitochondrial membrane potential changes and the release of cytochrome $\mathrm{C}$ by modulating the outer mitochondrial membrane permeability. Moreover, changes in protein kinase activity are observed during apoptosis in a variety of cell types indicating that phosphorylation is involved in cell death regulation. ${ }^{[3]}$ Physiologically, high doses of caffeine can result in inhibition of sodium reabsorption in the kidneys, thus preventing water re-absorption and causing diuresis. Studies reported an increase in urine production after ingestion of caffeine equivalent to $3-6$ cups of coffee ${ }^{[4]}$

Annaxin A5: a protein that is generally better known by its former name, annexin $\mathrm{V}$ binds to phospholipids in calcium-dependent manner and forms two-dimensional crystal lattices over the phospholipids bilayers that express phosphatidylserine .AnxA5 has become a widely used marker for detecting apoptotic cells because phosphatidylserine, which is normally localized within the internal leaflets of cytoplasmic membranes and is expressed on the cell surface during programmed cell death .Annexin A5 (or annexin $\mathrm{V}$ ) is a cellular protein of the annexin family. Annexin A5 has been proposed to play a role in the inhibition of blood coagulation by competing for phosphatidylserine binding sites with prothrombin, and also to inhibit the activity of phospholipase $A 1^{[5]}$ The aim of the study is to evaluate the effect of different doses of caffeine on the structure of submandibular salivary glands of adult male albino rats and to detect the degree of apoptosis immunohistochemically by using Annexin A5 marker.

\section{MATERIAL AND METHODS}

30adult male albino rats weighting (150$200 \mathrm{~g}$ ) were used in this study. The rats were maintained on stock diet and kept under fixed appropriate conditions of housing and handling. All experiments were carried out in accordance with the research Protocols established by The Animal Care Committee of TheNational Research Center (Cairo, Egypt), which followed the recommendations of The National Institutes of Health Guide for Care and Use of Laboratory Animals. Animals in each group were caged in separate cages in the animal house at The Faculty of Medicine, Sohag University.

\section{Materials}

The chemical used was caffeine Purity 99\% NT. it is powder in most company in Egypt under the license of Ajinomoto co.INC.Tokyo, Japan. The experimental doses selected according to (Leshchenko et al., 2012) ${ }^{[6]}$

\section{Experimental design:}

30 male albino rats were divided randomly into five equal groups $(n=6)$ control group: 6 rats were injected with sterile water intra peritoneally:

Group II \& IIa : 12 rats were intra pertoneally. Injected with single daily dose $(2.5 \mathrm{mg} / 100 \mathrm{~g}$ bodyweight/day) for three months this dose is equivalent to minimum human dose. 
Group IIa: 6 rats stopped treatment and left for one month after the period of treatment.

Group III \& IIIa: 12 rats were intra pertoneally. Injected with single daily dose $(10 \mathrm{mg} / 100 \mathrm{~g}$ body weight/day) for three months this dose is equivalent to maximum human dose.

Group IIIa: 6 rats stopped treatment and left for one month after the period of treatment

\section{Preparation of caffeine}

A stock solution was prepared by dissolving $400 \mathrm{~m} \mathrm{~g}$ of caffeine powder in $100 \mathrm{ml}$ of distilled water to obtain the dose of 2.5 and $10 \mathrm{mg} / \mathrm{ml}$ respectively. The dose schedule was so adjusted that the amount of caffeine administration per animal was as per their respective weight.

At the end of the experiment, the animals were $y$ sacrificed under anesthesia. Then the submandibular salivary glands were dissected out and immediately immersed in $10 \%$ formol saline for 48 hours to be processed and embedded in paraffin. The samples then were dehydrated in ascending grades of alcohol and then cleared in xylol. The tissues were impregnated with melted soft paraffin wax. Then embedded in hard paraffin. $4 \mu \mathrm{m}$ thick paraffin sections were obtained and mounted on clean glass slides andstained with Haematoxylin and Eosin (H\&E). ${ }^{[6]}$

\section{Immunohistochemical technique}

Immunohistochemical staining was done using Annexin A5 monoclonal antibody Paraffin were sectioned by microtome at $5-\mu \mathrm{m}$ thickiness. Sections were mounted on the positively charged glass slides .The slides were incubated at $37 \mathrm{c}$ over night for accurate adhesion of the section of the slide.Section were deparaffinization and rehydration by two changes .Slides were placed in xelene over night. And transferred into graded ethanol (100\%, 95\% and 70\%) 5 minutes each. Then washed in distilled water for 2 minutes then in phosphate buffer saline (PBS) for 5 minutes.Blocking endogenous proxidase activity: By incubated the slides in a solution of $30 \%$ hydrogen peroxide in $(180 \mathrm{ml}$ methyl alcohol +20 $\mathrm{ml} \mathrm{H} 2 \mathrm{O} 2$ ) for 30 minutes, this solution was freshly prepared. Slides were washed again in (PBS) for 5 minutes.

Antigen retrieval: This technique was used to unmask the antigen,as formaline fixation of tissue masks many antigens by forming intermolecular cross-links between formalin and antigens.Slides were placed in aglass container filled with sufficient target retrieval solution $180 \mathrm{cc}$ of distilled water $+20 \mathrm{ml}$ of target retrieval (Dako, Denmark). And treated in amicrowave oven over 100c for three successive trades, 5 minutes each. The amount of the fluid in the container was checked and was added if necessary to prevent slides from drying out. The container was removed from the oven and allowed to cool for 30 minutes. Slides were then washed in PBSphosphate buffer solution for 5 minutes. Application of primary antibody: The antibody was used in appropriate dilution (1:50), using PBS as adiluent and one to two dropes of the diluted primary antibody were added on each section. The slides were left in arefrigerator over night at $4 \mathrm{c}$. The anti-mouse annexin $\mathrm{v}$ monoclonal antibody was obtained from Medico company used in adilution of 1:50.The universal immunostaning detection kit (Dako Cytomation EnVision+Dual link system, Peroxidase (DAB+) Code K4065). It contains the following reagents and material. Labelled polymerHRBDAB+Substrate buffer (substrate buffer solution, $\mathrm{PH} 7,5$, containing hydrogen peroxide and apreservative). DAB +Chromogen (3,3-diaminobenzidine chromogen solution). The slides were incubated with the labeled polymer for 45 minutes. And rinsed in PBS for 5 minutes ,dried. Staning is completed by 5-10 minutes incubation with 3,3- diaminobenzidne (DAB)+Sustrate-chromogen which results in abrown-colored precipitate at the antigen site. washed in distilled water for 5 minutes

Counter stain: Slides were immersed in Mayor,s hematoxilline for 1 minute. washed in tap water andplaced in $70 \%, 95 \%$ and then $100 \%$ alcohol each 
for 5 minutes.The cover slides were mounted using DBX (Purified Canada balsam).

\section{Image analysis}

Sections stained PCNA were morphometricallyanalyzed using LEICA Quin 500 analyzer computer. AnnexinA5 immunostaining was measured as area $\%$ in astandard measuring frame in ten fields in each groupusing magnification (x400) by light microscopy transferred to the screen. Mean value and standard deviation were calculated for each specimen.

\section{Statistical analysis}

The Statistical Program SPSS for Windowsversion 19 was used for data entry and analysis. Graphics were done by Excel Microsoft office2013. Quantitative data were presented by meanand standard deviation. Novel Test wasused to compare more than two means in different groups. The lowest accepted level of significance was 0.05 or less.

\section{RESULTS}

I n H \& E stained sections, Submandibular gland of control group (I) was formed of parenchyma in the form of secretory end piece and collecting ducts. The secretory end pieces were formed of serous acini arranged in spherical fashion .each acinus was formed of pyramidal shaped serous cells withbasally located rounded deeply basophilic nuclei. The intercalated duct formed of cuboidal cells with narrow lumen and the .Striated ducts formed of low columnar highly acidophilic cells cells with centrally placed nuclei . The connective tissuestroma divided the gland into lobes and lobules andwas very delicate (Fig.,1 A) . In group II Acini appeared shrunken and their cytoplasm was vacuolated. Many nuclear changes appeared in form of (large, hyperchromatic, pleomorphic and pykontic) nuclei, also many mitotic Figure were observed. blood vessels appeared congested (Fig.,1 B) . In group IIa , there were decrease in the cytoplasmic vaccuolation of both secretory and duct system. No shirkage of acini was observed (Fig.1
C). In group III submandibular gland revealed loss of normal glandular architecture, degenerated serous acini, .destruction of basement membrane of the striated duct with dilated striated ducts and vacuolated cytoplasm of their cells. hyperchromatic nuclei (Fig.2 A) . In group IIIa degenerated serous acini as the prevous group . less dilated striated ducts were observed (Fig. 2 B).

\section{Masson - trichrome stain for collagen fibers :}

Submandibular glands of the control group showed thin capsule and very fine collagen fibers in between lobules and around the acini and (Fig. 3 A\&B).

In group II, there mild increase in collagen fibers in the capsule, around acini, around the ducts and between lobules (Fig. 3 C\& Fig. 4 A). In group II a, There was mild decrease in collagen fibers around acini and duct system compared to group II (Fig. 10). In group III, marked increase in collagen fibers in capsule in and around the ducts compared to control and previous groups (Fig. 4 B\&C). In group III a There was decrease in the amount of collagen fibers in compared to groupIII but still more the control (Fig. 5 A\& B).

\section{Annexin A5 immunostaning results}

Immuno-histochemical examination of control group of the submandibular glands showed no antibody reaction in either ducts or acini (Fig., 6 A). In group II showed weak positive reaction in the cytoplasm of the acini and strong positive reaction in the cytoplasm membrane of the duct system. While in groupIII showed high positive reaction in the cytoplasm of the acini and moderate positive reaction in the cytoplasm of the ducts (Fig., 6 B). In groupII a showed moderate positive reaction in duct system, and the cytoplasm of the acini showed very week positive reaction (Fig., $6 \mathbf{C}$ ). In group III, Marked increase in the positive reaction both in ducts and acini compared to the control (Fig., 7 A). While in group III a showed strong positive reaction in the cytoplasm of the acini and moderate reaction in the cytoplasm of the ducts (Fig., 7 B ) 


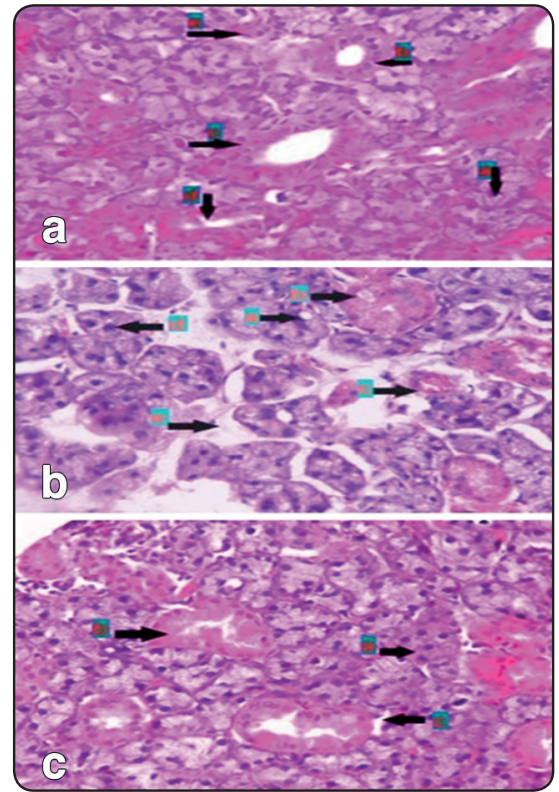

Fig. (1) (A): Aphotomicrograph ofcontrol submandibular salivary gland showing, serous acini (a) striated duct (b). interlobular duct (c) and intercalated duct (d) blood vessels (e) (H\&EX400). (B): photomicrograph s of submandibular salivary gland of the experimental Group II showing shrunken serous acini cells with vacuolated cytoplasm(a) vacuolated cytoplasm of striated duct cells (b) congestion of blood capillaries (c) hyperchromatic nuclei (d). with Increase space between acini (e) and (arrow). (H \& E X 400) (C): A Photomicrograph section of submandibular salivary gland of experimental groupIIa showing less vacuolated cytoplasm of serous acini cells comparedto the treated one (a) less vacuolated striated ducts (b). decrease interacinar spaces (c) (H \& E X 400)

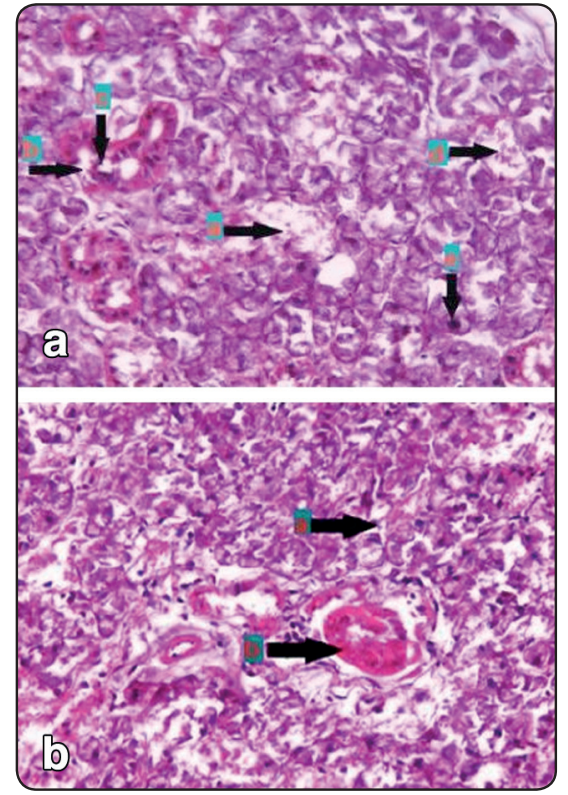

Fig. (2) (A): Aphotomicrograph of submandibular salivary gland of the experimental Group III showing degenerated serous acini (a),(d). destruction of basement membrane of the striated duct (b) with dilated striated ducts and vacuolated cytoplasm of their cells (c). hyperchromatic nuclei (e) (H \& E X 400). (B): Aphotomicrograph of submandibular salivary gland of the experimental group IIIa showing degenerated serous acini as previous group (a) less dilated striated ducts (b).( H \&E X 400)

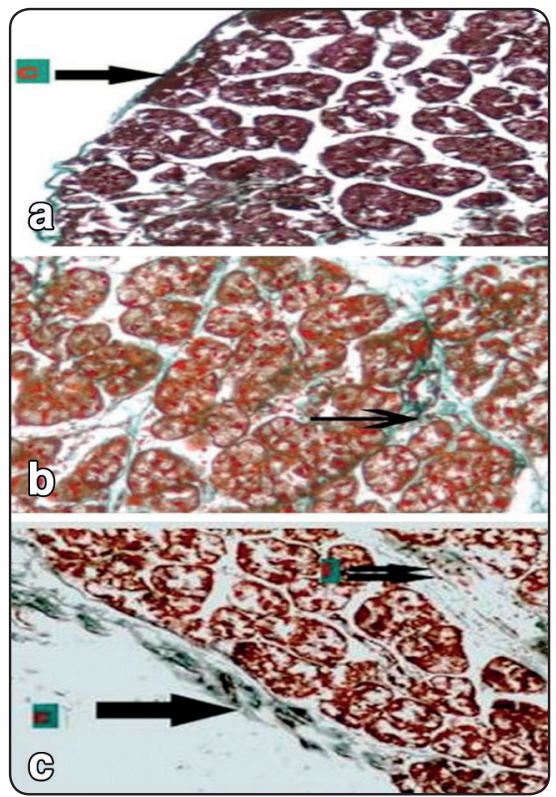

Fig. (3) (A): Aphotomicrograh of the control submandibular salivary gland group (1) showing thin capsule of the glands (arrow) (Masson trichrome $\mathrm{x} 400$ ) (B): Aphotomicrograh of the control submandibular salivary gland group (1) showing thin strands of collagen fibers (arrow) (Masson trichrome x400) (C): Aphotomicrograph of submandibular salivary gland of the experimental group II showing increase in collagen fibers in the capsule (c) and in between lobules (double arrow) (Masson trichrome x 400). 


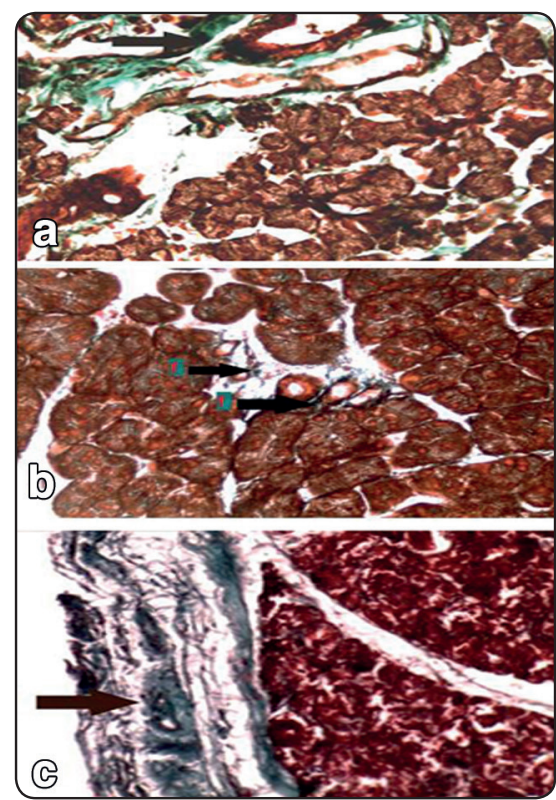

Fig. (4) (A): Aphotomicrograph of submandibular salivary gland of the experimental group II showing increase in collagen fibers around duct system (arrow) (Masson trichrome x400) (B): Aphotomicrograph of submandibular salivary gland of the experimental group IIa showing decrease in collagen fibers around duct system(f) (Masson trichrome x400) (C): A photomicrograph of submandibular salivary gland of the experimental group IIIshowing marked thickening of the capsule (arrow) and around duct system (f) (Masson trichrome $\mathrm{x} 400$ ).

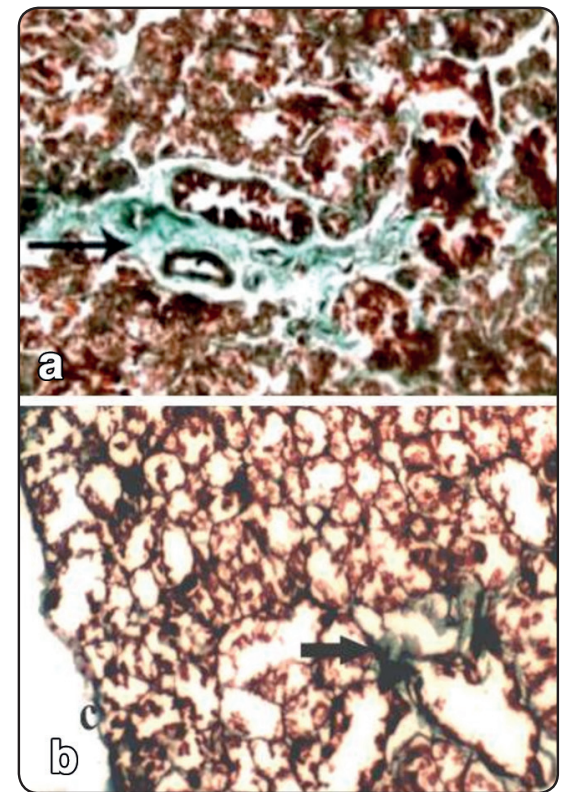

Fig. (5) (A): A Photomicrograph of submandibular salivary gland of the experimental group III showing marked increase in collagen fibers around duct system (arrow) (Masson trichrome x400). (B): A Photomicrograph of submandibular salivary gland of the experimental group IIIa showing few collagen fibers in the capsule (c) and $\mathrm{s}$ around duct system (arrow) (Masson trichrome $\mathrm{x} 400$ ).

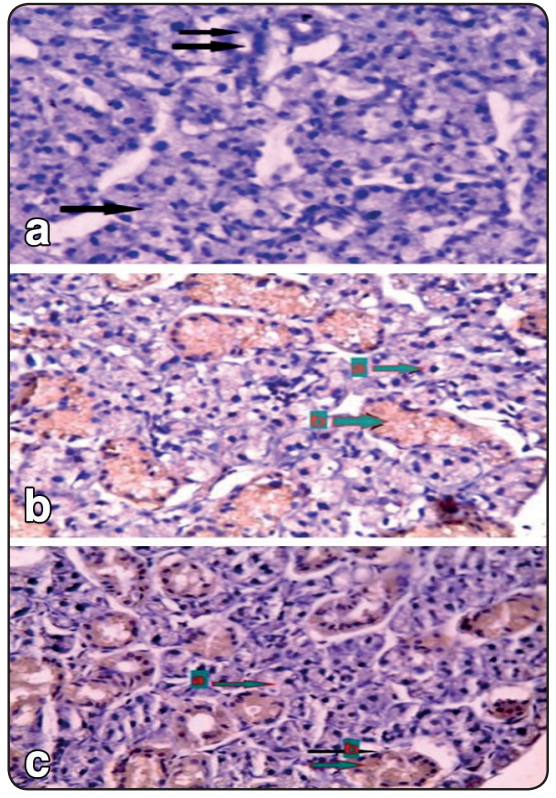

Fig. (6) (A): A Photomicrograph of control submandibular Saivary glands group (1) showing no antibody reaction in either ducts (double arrow) or in the acini (arrow) (Immunohistochemistry for Annexin v x 400). (B): Aphotomicrograph of the submandibular salivary gland of the experimental group II showingweak positive reaction in the cytoplasm of the acini (a) and strong positive reaction in the cytoplasmic basement membrane of the duct system (b) (Immunohistochemistry for Annexin $\mathrm{v}$ x400. (C): Aphotomicrograph of the submandibular salivary gland of the experimental group IIa showing positive reaction decreased in duct system(b) than groupIII (Immunohistochemistry Annexin v x400). 


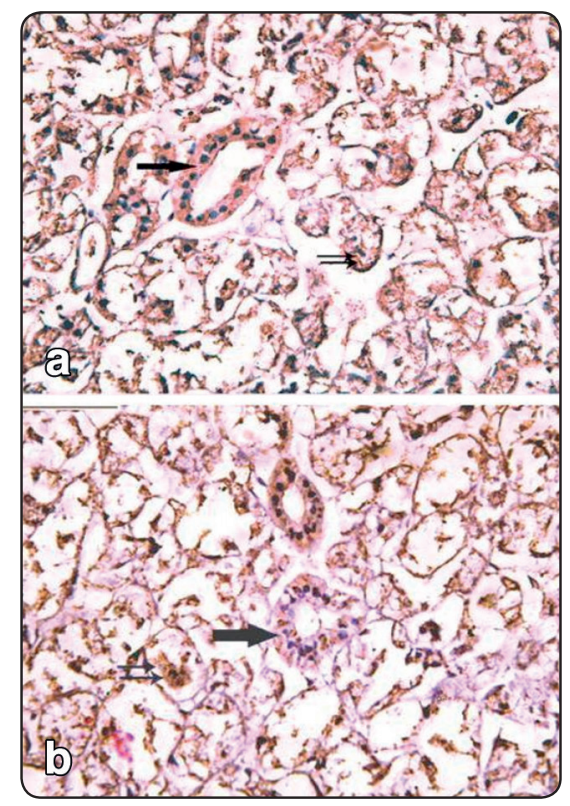

Fig.(7) (A): Aphotomicrograph of the submandibular salivary gland of the experimental group III showing high positive reaction in the cytoplasm of the duct system (Thick arrow) than cytoplasm of acini (double arrow) (immunohistochemistry Annexin v x400). (B): Aphotomicrograph of the submandibular salivary gland of the experimental group III showing moderate positive reaction in the cytoplasm of the duct system (Thick arrow) than cytoplasm of acini (Double arrow) (immunohistochemistry Annexin v x400).

Statistical analysis results (Table. 1 Fig. 8)

There is a highly significant statistical difference between all groups as regards annexin surface area $\%$ ( $\mathrm{p}$ - value $<0.0001$ ) where group III recorded the highest mean \pm SD values $(7.77( \pm 1.50))$ followed by group IIIa with mean \pm SD values of (22.78 $( \pm 1.39)$ while group1 recorded the lowest mean \pm SD values $(6.69( \pm 1.98))$.

TABLE (1) Comparison between mean values of annexin surface area $\%$ of all groups

\begin{tabular}{|c|c|c|c|c|c|c|}
\hline Area percentage & $\begin{array}{c}\text { Group I } \\
\mathrm{N}=6\end{array}$ & $\begin{array}{c}\text { Group II } \\
\mathrm{N}=6\end{array}$ & $\begin{array}{c}\text { Group IIa } \\
\mathrm{N}=6\end{array}$ & $\begin{array}{c}\text { Group III } \\
\mathrm{N}=6\end{array}$ & $\begin{array}{c}\text { Group III a } \\
\mathrm{N}=6\end{array}$ & $\mathrm{p}$ - value \\
\hline Mean (SD) & $6.69( \pm 1.98)$ & $12.25( \pm 4.34)$ & $7.77 \pm(1.50)$ & $29.37( \pm 5.15)$ & $22.78( \pm 1.39)$ & $<0.0001$ \\
\hline
\end{tabular}

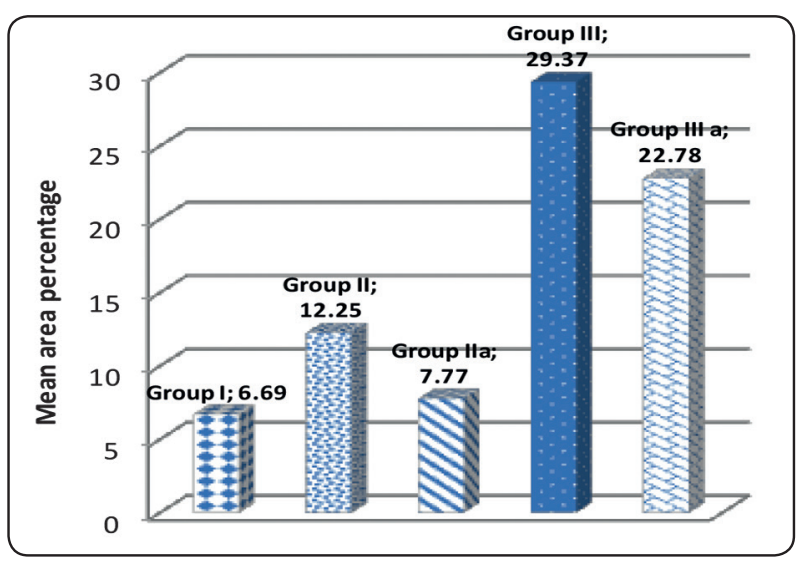

Fig (8) Area percentage in different groups.

\section{DISCUSSION}

Caffeine is a central nervous system stimulant with diuretic properties. It may reduce salivary flow by direct effects upon the salivary glands through effects on the autonomic nervous system or through diuresis and dehydration ${ }^{[7]}$. There were varying changes in the glandular architecture including changes in the acini, ducts, connective tissue stroma and blood vessels. These changes varied from one group to another according to the dose administered $^{[8]}$. There was cytoplasmic vacuolation as an effect of energy drinks on submandibular 
glands. Caffeine is one of the main ingredients of energy drinks may led to degenerative changes within the secretory portions that mostly of (fatty degeneration), these finding in accordance with previous studies ${ }^{[9]}$. The nuclei of the secretory cells showed nuclear atypism, pleomorphism and hyperchromatism. Numerous mitotic figures were also detected. All these findings were considered premalignant. Dilatation and congestion of the blood vessels in between the acini might be attributed to the microcirculatory disturbances that developed as side effect of caffeine ${ }^{[10]}$. The spaced serous acini could be attributed to edema ${ }^{[11]}$ In the present study, administration of caffeine in a dose of $(10 \mathrm{mg} / \mathrm{kg}$ b.w) induced sever histopathological alterations in the gland. Light microscopic examination revealed marked degenerative changes compared to the groups received caffeine in a dose $(2,5 \mathrm{mg} /$ $\mathrm{kg}$ b.w). Hydropic degeneration is a disturbance in the metabolism of the cell resulting in morphologic abnormalities. Thus, the cells could lose their normal architectural pattern of arrangement ${ }^{[12]}$. The dilatation of the ducts in the present study occurred due to glandular damage and dysfunction caused by caffeine administration. These finding coincide with a previous study. Which clarified that the duct dilatation in the submandibular glands of advancing aged mice occurred whenever the gland was atrophied or there was impairment in its function. ${ }^{[9]}$ vacuolization in response to injury is fully reversible, we observed that administration of caffeine in small doses could be reversed while the changes due to large doses of caffeine could not. The empty spaces left by detached and lost a cinar cells is supposed to be occupied by undifferentiated progenitor cells migrating from the intercalated duct compartment to the a cinar cell compartment ${ }^{[13,14]}$.In the present study there was a increase of collagen fibers in all groups treated with of caffeine these finding in agreement with some authors who reported that thickening of the connective tissue capsule and extensive fibrosis of the connective tissue septa were detected in the submandibular salivary glands received red bull energy. Excessive fibrosis might be due to toxic effect of caffeine, ${ }^{[9]}$ In group III which received caffeine in a dose of $(10 \mathrm{mg} / \mathrm{kg}$ b.w $)$ there was marked thickening of the capsule, increase collagen in between lobules and around acini compared to group II these finding agree with some previous studies ${ }^{[15]}$. In the present study, in groups stopped caffeine treatment, collagen decreased compared to the treated groups and this is an agreement with previous studies hich revealed that all extensive injuries even fibrosis could recover after prolonged period of caffeine administration. ${ }^{[16]}$ In the present study apoptosis was detected by (Annexin A5). There were progressive increase in apoptotic marker with the increase of the caffeine dose. The apoptotic marker attenuated after stopping caffeine treatment. The immune histochemistry finding agree with ${ }^{(17)}$ who reported that exposure of caffeine induces various cell responses including cell death. We further investigated whether caffeine-induced cell death represents apoptosis or necrosis. Apoptosis Is Induced in bone Cells by low Concentrations of Caffeine. The phosphatidylserine exposure on the surface of apoptotic cells is a general apoptosis feature occurring before membrane bleb formation and inter nucleosomal DNA cleavage. Annexin-v FITC is recently employed in evaluation and quantification of cellular apoptosis by flow cytometry due to its specific bind to phosphatidylserine residues. Thus, viable cells are classified as annexin $\mathrm{V}$ negative, while the apoptotic cells are annexin $\mathrm{V}$ positive In addition, the concomitant uses of nuclear fluorescent dye propidium iodide makes possible to verify the characteristic nuclear alterations of the late apoptosis stages, since the membrane permeability increases ${ }^{[18]}$. Cytotoxicity of caffeine may be due to its ability to trigger apoptosis A number of cysteine proteases, known as caspases, play important roles in apoptosis similar to members of the Bcl2 family which regulate mitochondrial membrane potential changes and the release of cytochrome $\mathrm{C}$ by modulating the outer mitochondrial membrane permeability. Moreover, changes in protein kinase 
activity are observed during apoptosis in a variety of cell types indicating that phosphorylation is involved in cell death regulation ${ }^{[19]}$.The renewal of apoptotic and or detached acinar cells is supposed to occur via recruitment of undifferentiated progenitor cells from the intercalated duct, which connects the a cinus with the more proximal striated duct This theory was recently supported by studies using cloned progenitor cells .Acinar cells may also, to an extent, divide in situ. ${ }^{[20]}$ from the obtained results we could concluded that, excessive use of Caffeine markedly affect the histological structure of submandibular salivary gland. These changes could be reversible after caffeine stoppage if the doses is small while the histopathological changes of the gland is irreversible if the doses is large. More deep studies are needed to clear the appropriate amount of caffeine that may be used without salivary affection.

\section{REFERENCES}

1- Frary CD, Johnson Rk \& Wang MQ(2005): Food sources and intakes of caffeine in the diets of persons in the United States .

2- AnanyaM. (2014):what is Caffeine?News-Medical.net - An AZoNetwork Site. http://www.news-medical.net/ health/What-is-Caffeine.aspx.

3- Fernandez MJ, Lopez A, Santa-Maria A. (2003) Apoptosis induced by different doses of caffeine on Chinese hamster ovary cells. J. Appl. Toxicol 23:221-224.

4- Bratton DL.(1997): Appearance of phosphatidylserine on apoptoticcells requires calcium-mediated nonspecific flip-flop and is enhanced byloss of the aminophospholipid translocase. J. Biol. Chem. 272:26159-26165.

5- Koopman G., Reutelingsperger, C.P., Kuijten, G.A.M. (1994). Annexin V for flow cytometric detection of phosphatidyl serine expression on B cells undergoing apoptosis. Blood 84, 1415-20. 2 Cancer.;18:1007-1021

6. Meyer HE, Pedersen JI, Loken EB, Tverdal A. (1997): Dietary factors and the incidence of hip fracture in middleaged Norwegians. A prospective study. Am. J. Epidemiol. 145:117-123.

7- Lee N, MacDonald H, Reinhard C, Halenbeck R, Roulston A, Shi T, Williams LT. (1997) : Activation of hPAK65 by caspase cleavage induces some of the morphological and biochemical changes of apoptosis.Proc. Natl. Acad. Sci. U S A.; 94:13642-13647.

8- Ugwuja, E (2014): Biochemical Effects of Energy Drinks Alone or in Combination with Alcohol in Normal Albino Rats. Advanced Pharmaceutical Bulletin ,4(1), 69-74.

9- Mubarak, R. (2012): Effect of red bull energy drink on Rat's submandibular salivary glands (Light and Electron microscopic study). J. Amer. Sci. 8(1):366-372.

10- Barceloux D.G., Bond G.R., Krenzelok E.P. Cooper H \& Vale J.A. (2002): American Academy of Clinical Toxicology Practice Guidelines on the Treatment.

11- McKinnell I.W., Rundnicki M.A.(2004):Molecular Mechanisms of Muscle Atrophy. Cell,119, 907.

12- Abdin F, (1981): Cell and Tissue Damage. In Abdin's General Pathology (4 ${ }^{\text {th }}$ ed., p. 7).

13. Xia Z, Dickens M, Raingeaud J, Davis RJ, Greenberg ME. (1995): Opposing effects of ERK and JNK-p38 MAP kinases on apoptosis. Science. 270:1326-1331.

14- Laine M, Virtanen I, Salo T, Konttinen YT.(2004): Segment-specific but pathologic laminin isoform profiles in human labial salivary glands of patients with Sjogren's syndrome. Arthritis Rheum; 50.

15- Ruhl CE, Everhart JE (2005): Coffee and caffeine consumption reduce the risk of elevated serum alanine aminotransferase activity in the United States. Gastroenterology. ;128:24

16- Menezes S.L., Bozza P.T., Neto H.C., Laranjeira A.P., Negri E.M., Capelozzi V.L., Zin W.A.(2005): Pulmonary and Extra Pulmonary Acute Lung Injury. J. Applied. Physiol., 98(5), 1777-1778.

17- He, Z.; Ma, W. Y.; Hashimoto, T.; Bode, A. M.; Yang, C. S.; Dong, Z. (2003):Induction of apoptosis by caffeine is mediated by the $\mathrm{p} 53$, Bax, and caspase 3 pathways. 63, 4396-4401.

18- Chaves M.M., Kallas E.G.(2004):Cell cycle distribution of CD4+lymphocytes in HIV-1-infected subjects. Cytometry; 62B:46-51.

19- Hsia, J. Y., Chen, C. Y., Hsu, C. P., Shai, S. E., Yang, S. S., Chuang, C.Y., Wang,P.Y., and Chen, J.T.(2001):Expression of apoptosis-regulating proteins p53, Bcl-2, and Bax in primary resected esophageal squamous cell carcinoma. Neoplasma, 48: 483-488.

20- Kishi T, Takao T, Fujita K, Taniguchi H (2006). Clonal proliferation of multipotent stem/progenitor cells in the neonatal and adult salivary glands. Biochem Biophys; 340:544-52. 